\title{
ULTRA-SHORT ELECTRON BUNCH GENERATION WITH A RECTILINEAR COMPRESSOR
}

\author{
L. Serafini, A. Bacci, INFN-Milan, Via Celoria 16, 20133 Milan, ITALY \\ M. Ferrario, INFN-Frascati, Via E.Fermi 33, 00144 Frascati, ITALY
}

\begin{abstract}
We describe a new method for reducing the electron bunch length of high brightness beams in linacs, as those required to drive X-ray SASE FEL's, which is of interest also for advanced accelerator applications like plasma accelerators, where low charge femtosecond bunches are requested. The method is based on a rectilinear compressor scheme utilizing the bunching properties of RF slow waves. It is shown that whenever a beam, slower than the synchronous velocity, is injected into a RF wave at zero acceleration phase and slips back in phase up to the peak acceleration phase, it can be longitudinally compressed even when already relativistic (tens of MeV). This occurs if the beam is extracted from the wave at the synchronous velocity: in this case the bunch undergoes a quarter of synchrotron oscillation that induces a phase compression up to a factor of 20 when proper care is taken to preserve the longitudinal emittance. Compression is actually not limited by space charge effects but by RF non-linearities in the longitudinal phase space, that produce a longitudinal emittance growth. A few examples are presented to demonstrate the potentialities of this method, by which multi-kA beams with very low transverse emittance can be generated at moderate energies (about 100 $\mathrm{MeV})$.
\end{abstract}

\section{INTRODUCTION}

The need to produce high brightness electron beams delivered in short sub-ps bunches has been driven by the demands of X-ray SASE FEL's, which need multi-GeV beams with multi-kA peak currents and bunch lengths in the 100-300 fs range, associated to normalized transverse emittances lower than $1 \mathrm{~mm}$ mrad. Present designs of such FEL's are based on the use of photo-injectors in conjunction with magnetic compressors. Since the impact of magnetic compressors on the beam quality is quite relevant, with tendency to cause a serious emittance growth due to Coherent Synchrotron Radiation effects in their bends, we explored a new method able to compress the bunch at moderate energies (tens of $\mathrm{MeV}$ ) on rectilinear trajectories, which is based on the weak synchrotron motion that the electron beam still undergoes at those energies in the RF wave of the linac accelerating structures: this residual synchrotron motion can be enhanced by using slow waves.

We show here how this method can preserve the low transverse emittance achieved at the exit of the photoinjector, reaching at the same time peak currents comparable with those produced by magnetic compressors.

\section{VELOCITY BUNCHING IN RF WAVES}

The interaction of a beam with a RF wave given by $E_{z}=-E_{0} \sin \left(\omega t-k z+\psi_{0}\right)$ is described by an Hamiltonian $H=\gamma-\beta_{r} \sqrt{\gamma^{2}-1}-\alpha \cos \xi$, where the normalized electron energy is $\gamma=1+T / m c^{2}$, $\xi=k z-\omega t-\psi_{0}$ is its phase with respect to the wave, while $\alpha \equiv e E_{0} / m c^{2} k$ is the dimensionless vector potential amplitude and $\gamma_{r}=1 / \sqrt{1-\beta_{r}^{2}}$ is the resonant gamma of the wave (conventional traveling wave structures operate at $\left.\beta_{r}=1, k=\omega / \beta_{r} c\right)$. A wave whose phase velocity is slightly smaller than $c$, so that $k=\omega / c+\Delta k$, is characterised by $\beta_{r}=1-c \Delta k / \omega$, $\gamma_{r}=\sqrt{\omega / 2 c \Delta k} \quad$ (assuming $c \Delta k / \omega<<1$ ).

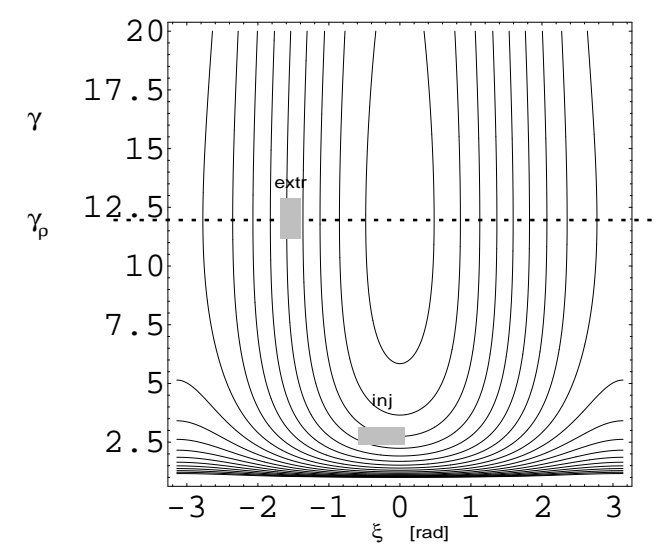

Fig.1 Phase space plots of a slow RF wave ( $\gamma_{r}$ finite) showing the basics of phase compression in a linac

The basic mechanism underlying the rectilinear compression effect is illustrated in Fig.1, where the contour lines of the Hamiltonian associated to a slow RF wave having $\gamma_{r}=12$ (i.e. $\beta_{r}=0.9965$ and $\Delta k=0.0035 \omega / c$ ) and $\alpha=0.2$ are plotted. If the bunch is injected into the wave at zero phase (i.e. when the field of the wave is not accelerating) at an energy lower than the synchronous one (which corresponds to $\gamma_{r}$ ), it will slips back in phase and go up in energy (being accelerated by the wave). By extracting the beam from the wave at the time it reaches the resonant $\gamma_{r}$, i.e. when it becomes synchronous with the wave, we make the bunch undergo one quarter of synchrotron oscillation. 
In doing that the beam is compressed in phase as depicted in the figure.

The compression ratio has been calculated elsewhere [1] to be $C=2 \delta \psi_{0}\left|\sin \bar{\xi}_{e x}\right| / \sqrt{\delta \psi_{0}^{4}+\left(\frac{1}{\alpha \bar{\gamma}_{0}} \frac{\delta \gamma_{0}}{\bar{\gamma}_{0}}\right)^{2}}$, where $\delta \psi_{0}$ and $\delta \gamma_{0} / \bar{\gamma}_{0}$ are the initial phase spread and energy spread of the bunch $\left(\bar{\xi}_{e x}\right.$ is the average beam exit phase at $\gamma=\gamma_{r}$ ). This expression gives a good first order estimate of the compression for an uncorrelated longitudinal phase space distribution at injection. The actual beam produced by photo-injectors has energy-phase correlations given by space charge and RF effects, so that the best performances of a rectilinear RF compressor may occur for extraction of the beam from the wave at energies different than $\gamma_{r}$, as shown in the following.

\begin{tabular}{|l|l|l|}
\hline$\gamma_{r}$ & $\beta_{r}$ & $\Delta k$ \\
\hline 20 & 0.9987 & 0.0013 \\
\hline 35 & 0.9996 & 0.0004 \\
\hline 60 & 0.99986 & 0.00014 \\
\hline infinite & 1 & 0 \\
\hline
\end{tabular}

Table 1: Resonant gamma's and corresponding phase velocities and wave number shifts

In order to illustrate this property we show results of a simulation performed on a beam produced by a S-band photo-injector at $7 \mathrm{MeV}(1 \mathrm{nC}$ bunch charge) with $100 \mathrm{~A}$ peak current, which is injected into a wave extending indefinitely in the $\mathrm{z}$ direction, at 4 different values of $\gamma_{r}$, as reported in Table 1. The accelerating gradient at S-band was taken $5 \mathrm{MV} / \mathrm{m}$, corresponding to $\alpha=0.16$.

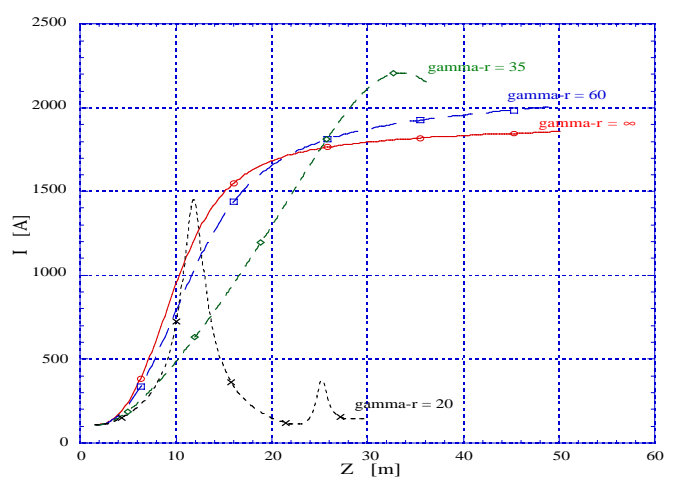

Fig.2 Peak current in the bunch as a function of the distance of interaction with the RF wave

In Fig. 2 it is shown the behavior of the peak current, that can reach up to $2 \mathrm{kA}$ after $30 \mathrm{~m}$ of interaction length, with an optimum value for the resonant gamma $\gamma_{r}=35$. The bunch energy $(45 \mathrm{MeV})$ is at this point much larger than $\gamma_{r}$, probably due to the need to overcompensate the correlated energy spread of the bunch at injection (as shown later in Fig.6). It is worth noticing that the longitudinal phase space at the point of maximum current $(\mathrm{z}=32 \mathrm{~m})$ is strongly non-linear due to the rotation in the wave potential, as shown in Fig.4. The current histogram reveals that the current distribution is also nonuniform, with a sharp peak on the bunch head that reaches currents in excess of $5 \mathrm{kA}$ (see Fig.5), in comparison to an average current of $2 \mathrm{kA}$.

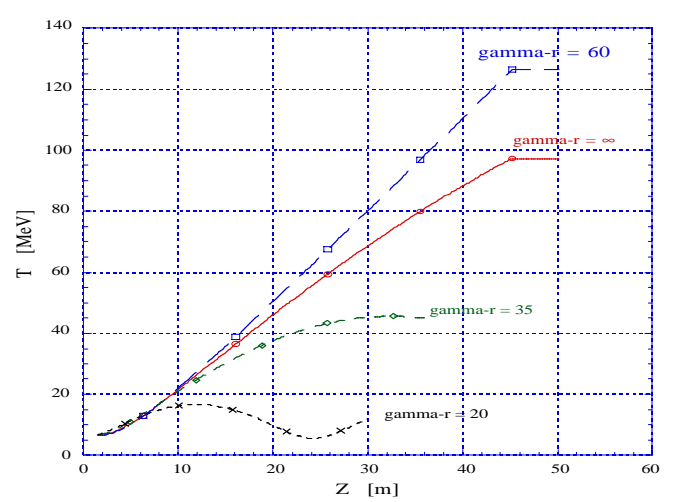

Fig.3 Average kinetic energy of the bunch for same parameters as for Fig.2

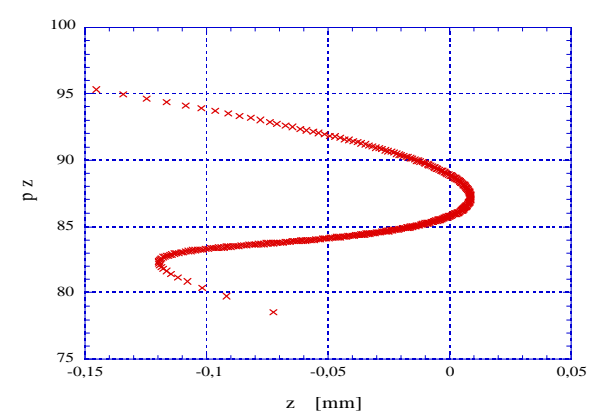

Fig.4 Longitudinal phase space distribution at the maximum peak current position

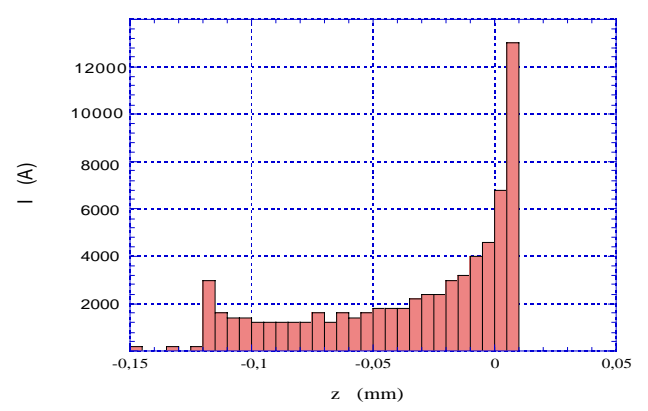

Fig.5 Current distribution in the bunch

In order to preserve the beam transverse emittance we have to integrate the longitudinal dynamics of the RF compressor with the process of emittance compensation, which is achieved for a beam at constant current by matching it on the invariant envelope. In our case the envelope equation, assuming a current increasing like the 
energy, $\quad I=I_{0} \gamma / \bar{\gamma}_{0} \quad, \quad$ becomes $\sigma^{\prime \prime}+\gamma^{\prime} \sigma^{\prime} / \gamma+\Omega^{2} \gamma^{\prime 2} \sigma / \gamma^{2}-I_{0} / 2 I_{A} \bar{\gamma}_{0} \sigma \gamma^{2}=0$.

The equivalent expression for the invariant envelope [2], i.e. an exact solution of the envelope equation, is in this case $\hat{\sigma}=\frac{1}{\Omega \gamma^{\prime}} \sqrt{\frac{I_{0}}{2 I_{A} \bar{\gamma}_{0}}}(\Omega$ is the dimensionless focusing frequency and $\gamma^{\prime}$ the normalised accelerating gradient), i.e. a parallel envelope, to be compared to the previous $\sigma_{I N V}=\sqrt{I_{0} / 6 I_{A} \gamma} / \Omega \gamma^{\prime}$, valid for constant current, and predicting an envelope scaling as $1 / \sqrt{\gamma}$. The matching requires in this case an additional focusing, for example by an external solenoid, as shown in Fig.6.

Here we apply velocity bunching within the lay-out of the LCLS injector [3], showing that the emittance correction process is altered but gives same performances as without the compressor, i.e. we can expect no significant emittance degradation.

As a last example of the velocity bunching, we show in Fig. 7 how a $30 \mathrm{fs}$ rms long electron bunch (at a charge of $16 \mathrm{pC}$ ) could be produced with the proposed ORION injector lay-out, enhanced by adding a $1 \mathrm{~m}$ S-band TW structure working at $\gamma_{r}=27$ between the S-band RF gun and the $\mathrm{X}$-band accelerating structures.

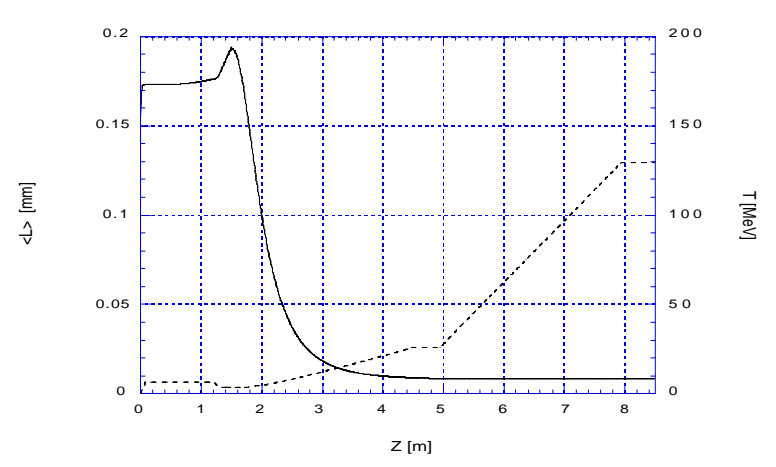

Fig.7 Velocity bunching with the ORION injector (bunch length, left scale in $\mu \mathrm{m}$, beam energy, right scale)

\section{REFERENCES}

[1] L.Serafini, M.Ferrario, Velocity Bunching in Photoinjectors, Proc. of ICFA Workshop on the Physics of and Science with the X-ray FEL, Arcidosso, Sept. 2000

[2] L.Serafini,J.Rosenzweig,Phys. Rev. E 55(1997) 7565

[3] M.Ferrario et al., HOMDYN Studies for the LCLS $R F$ Photoinjector, Proc. of ICFA Workshop on the Physics of High Brightness Beams, UCLA, Nov. 1999

[4] D.T.Palmer et al., The ORION Photoinjector, this conference
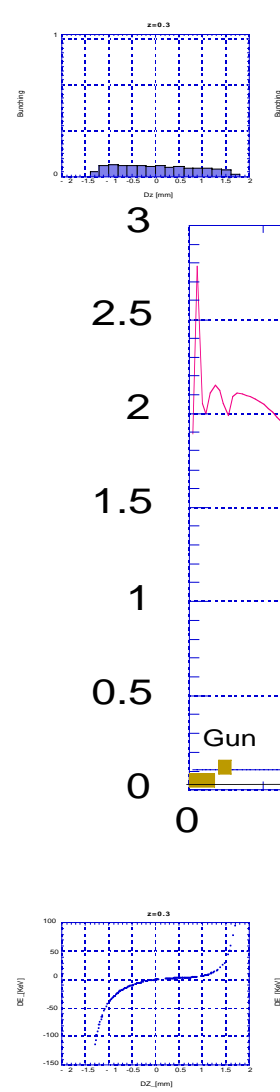
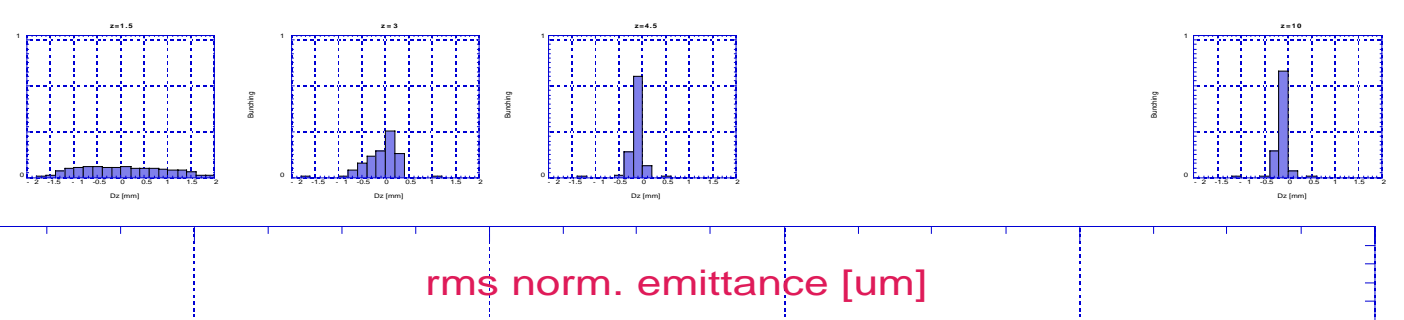

rms norm. emittance [um] beam current [kA]
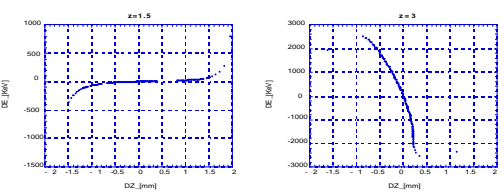

4
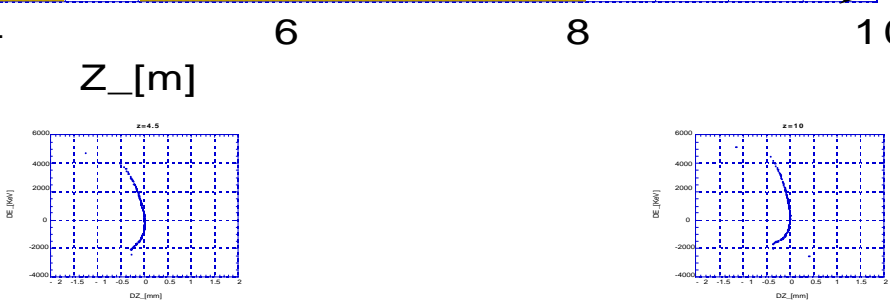

Figure 6 Example of rectilinear RF compressor using LCLS injector lay-out $\left(\gamma_{r}=27, \Delta k=7 \cdot 10^{-4}, \alpha=0.75\right.$ ), with full integration of the emittance correction process into the velocity bunching process 